\title{
Safety of Transcranial Direct Current Electrical Stimulation in Dermatomyositis: A Case Report
}

\author{
Rafael Giovani Missé1, Luiz Felipe Adsuara de Sousaㅁ, Lucas de Macedo dos Santos², \\ Abrahão Fontes Baptista ${ }^{2,3}$, Clarice Tanaka², Julia Maria D'Andréa Greve4, \\ Samuel Katsuyuki Shinjo ${ }^{*}$ (D)
}

\begin{abstract}
${ }^{1}$ Division of Rheumatology, Faculdade de Medicina FMUSP, Universidade de Sao Paulo, Sao Paulo, SP, Brazil (BR)
${ }^{2}$ Department of Physical Therapy, Speech Therapy and Occupational Therapy, Hospital das Clinicas HCFMUSP, Faculdade de Medicina, Universidade de Sao Paulo, Sao Paulo, SP, Brazil (BR)

${ }^{3}$ Center for Mathematics, Computing and Cognition, Universidade Federal de ABC, SP, Brazil (BR)

${ }^{4}$ Laboratory of Movement Studies, Institute of Orthopedics and Traumatology, Hospital das Clinicas HCFMUSP, Faculdade de Medicina, Universidade de Sao Paulo, Brazil (BR)

Email: *samuel.shinjo@gmail.com
\end{abstract}

How to cite this paper: Missé, R.G., de Sousa, L.F.A., de Macedo dos Santos, L., Baptista, A.F., Tanaka, C., D’Andréa Greve, J.M. and Shinjo, S.K. (2020) Safety of Transcranial Direct Current Electrical Stimulation in Dermatomyositis: A Case Report. Open Journal of Rheumatology and Autoimmune Diseases, 10, 88-93. https://doi.org/10.4236/ojra.2020.102011

Received: April 18, 2020

Accepted: May 26, 2020

Published: May 29, 2020

Copyright $\odot 2020$ by author(s) and Scientific Research Publishing Inc. This work is licensed under the Creative Commons Attribution International License (CC BY 4.0).

http://creativecommons.org/licenses/by/4.0/

\begin{abstract}
Transcranial direct current stimulation (tDCS) has emerged as a nonpharmacological tool in physical rehabilitation. There have currently no studies that evaluated the safety and efficacy of tDCS in patients with dermatomyositis. Case-report: Three adult women with dermatomyositis were allocated randomly to intervention (i-tDCS, one patient) or not (sham-tDCS, two patients) of three consecutive days of tDCS and evaluated in four periods: before-tDCS (PRE), 15 minutes after-tDCS (0th POST), 15 days after-tDCS (15th POST), and 30 days after-tDCS (30th POST). The tDCS was safe throughout the protocol, without disease relapsing or adverse effects related to tDCS. Furthermore, the tDCS increased the muscle torque and total work of dominant and non-dominant elbow flexors in the patient with i-tDCS, when compared to two patients with sham-tDCS. Conclusions: The tDCS was safe and appeared to influence long-term strength in the limb of the patient with stable dermatomyositis.
\end{abstract}

\section{Keywords}

Dermatomyositis, Neuromodulation, Safety, Systemic Autoimmune Myopathies

\section{Introduction}

Dermatomyositis (DM) is a systemic autoimmune myopathy characterized clin- 
ically by cutaneous and muscle inflammations [1]. The cutaneous lesions are represented by heliotrope rash and Gottron's papules, whereas the muscle involvement is expressed by progressive, symmetrical and predominantly proximal muscle weakness of the limbs, leading to significant disability and higher morbidity [1].

Currently, patients with systemic autoimmune myopathy have achieved health, quality of life and general clinical improvement with different pharmacological strategies (e.g., glucocorticoids, immunosuppressive and/or immunobiological drugs) and non-pharmacological therapies (e.g., exercise training) [1] [2] [3] [4]. However, the magnitude of clinical and laboratory progress is not always accompanied by the improvement of muscle strength and functionality [1] [3]. In this context, the maintenance of these parameters constitutes one of the main challenges in the treatment of systemic autoimmune myopathies. Therefore, strategies complementary to pharmacological and non-pharmacological therapies could promote additional effects on muscle strength and function.

Transcranial direct current stimulation (tDCS) is a noninvasive method that utilizes electrical stimulation in the scalp, leading to better motor rehabilitation [5] [6] [7] [8]. Thus, this method could be a valuable auxiliary therapeutic tool for maintaining strength in patients with several neurological disorders, such as cerebral palsy and Parkinson's disease [9]. Regarding rheumatic patients, better motor function is observed in patients with fibromyalgia [9] [10].

However, up to date, there have no tDCS studies in patients with DM. In contrast, encouraging results with tDCS have shown positive acute and chronic effects on isometric muscle strength in healthy individuals. Moreover, similar muscle strength improvement has been reported in post-stroke patients with motor sequelae in the upper and lower limbs. The objective of the proposed study was to assess: 1 ) the safety of tDCS, and 2) the effectiveness of tDCS on the ability to produce positive muscle strength in a patient with stable DM.

\section{Case Report}

Three sedentary women with DM (EULAR/ACR criteria) [1] were included and randomly allocated by an external health professional under the conditions tDCS-active or tDCS-sham. [1] was included at June 2019.

The patients were informed of the risks and benefits through a consent form. The patient submitted to the active tDCS at disease onset, she had expressive upper and lower limb muscle weakness with cutaneous lesions (heliotrope rash, Gottron's papules/signs, "V-neck" sign, "shawl” sign, periungueal telangiectasia with associated dystrophic cuticles), and received methylprednisolone and immunoglobulin human intravenous pulse therapies, oral glucocorticoids and different immunosuppressive drugs. Seven years after disease onset, she had been using only mycophenolate mofetil ( $2 \mathrm{~g} /$ day) with stable disease.

The anodal tDCS was applied with an electric current intensity of $2 \mathrm{~mA}$ and a density of $0.057 \mathrm{~mA} / \mathrm{cm}^{2}$ for 20 minutes. The electrodes were positioned ac- 
cording to the international system The positively charged electrode was positioned at $\mathrm{C} 1$ (contralateral to the dominant limb) and the negatively charged electrode was positioned in the supraorbital region FP2 (ipsilateral to the dominant limb). The tDCS-sham was performed through the same assembly followed by a 30 -second stimulation. During the session, the patient remained seated.

Considering the clinical relevance of muscle strength in these diseases, dynamic elbow strength was assessed through the isokinetic dynamometer (Biodex System 3 Pro) [2]. To avoid a learning effect, a previous familiarization was performed during baseline measurement, and the patient was given maximum encouragement.

The isokinetic dynamometer assessment was performed over four periods on the patient from the present study: 15 minutes before-tDCS (PRE), 15 minutes after-tDCS $\left(0^{\text {th }}\right.$ POST), 15 days after tDCS $\left(15^{\text {th }}\right.$ POST), and 30 days after tDCS $\left(30^{\text {th }}\right.$ POST).

The disease status was evaluated by the International Myositis Assessment and Clinical Groups Studies (IMACS) set scores [1] [3] [4]: Visual Analogue Scale (Physician and Patient), Myositis Disease Activity Visual Assessment Scales (MYOACT), Health Assessment Questionnaire (HAQ), Manual Muscle Testing (MMT)-8 and laboratory evaluation: aspartate aminotransferase (reference value: $>31 \mathrm{U} / \mathrm{L})$, alanine aminotransferase $(>31 \mathrm{U} / \mathrm{L})$, lactic dehydrogenase (240 $480 \mathrm{U} / \mathrm{L})$, creatine phosphokinase $(26-308 \mathrm{U} / \mathrm{L})$ analyzed by automated kinetic methods. The laboratory assessment was performed over three periods of tDCS: PRE, $15^{\text {th }}$ POST and $30^{\text {th }}$ POST. The data was analyzed by percentage changes from the base-line: $[\Delta$ (POST - PRE) $/ 100] \%$.

Before the first session low values of the IMACS set scores and preserved muscle strength assessed by MMT-8 (Table 1), a reduction of torque values was observed in relation to the dominant and non-dominant limb of the patient submitted to tDCS in comparison to the patients submitted to Sham: \#Patient (10.5) vs. Sham \#1 (26.7) and Sham \#2 (27.8) as well as, evidenced in relation to total work (J) (42.5) vs. (160.5) and (158.5).

After the first session, there was an increase in torque values in both limbs $(+29.5)$ in the patient who received the tDCS, when compared to Sham \#1 $(+17.6)$ and Sham \#2 $(+10.7)$. Moreover, the total work in the patient was +11.7 J, whereas in the Sham\# 1 and Sham \#2 were $+22.6 \mathrm{~J}$ and $+19.4 \mathrm{~J}$, respectively.

Interestingly, after 15 days there was a greater increase in the patient who received tDCS compared to Sham patients in both limbs, similar to the total work (Table 2).

In the final analysis, it was observed that the patient who underwent tDCS maintained the gains on torque of the flexors in the dominant limb, while, in relation to the non-dominant limbs, a reduction was observed. However, the reduction was smaller relative to one patient, while another showed increases in the final analysis. So it was observed in the total work. 
Table 1. General features of the patients before transcranial direct current electrical stimulation.

\begin{tabular}{|c|c|c|c|}
\hline & Case & Control \#1 & Control \#2 \\
\hline \multicolumn{4}{|l|}{ General data } \\
\hline Age (years) & 52 & 60 & 52 \\
\hline Height $(\mathrm{cm})$ & 1.50 & 1.63 & 1.69 \\
\hline Weight (kg) & 73 & 77 & 74 \\
\hline $\operatorname{BMI}\left(\mathrm{kg} / \mathrm{m}^{2}\right)$ & 24.3 & 29.0 & 25.9 \\
\hline Disease duration (days) & 1718 & 1482 & 1132 \\
\hline \multicolumn{4}{|l|}{ Disease status } \\
\hline HAQ $(0.00-3.00)$ & 0.00 & 0.00 & 0.00 \\
\hline MYOACT $(0-60)$ & 0 & 0 & 0 \\
\hline Patients' VAS $(0-10 \mathrm{~cm})$ & 0 & 0 & 0 \\
\hline Physician' VAS $(0-10 \mathrm{~cm})$ & 0 & 0 & 0 \\
\hline MMT-8 $(0-80)$ & 80 & 80 & 80 \\
\hline
\end{tabular}

BMI: body mass index. HAQ: Health Assessment Questionnaire; MMT: Manual Muscle Testing; MYOACT: Myositis Disease Activity Visual Assessment Scales: VAS: Visual Analogue Scale.

Table 2. Data related to peak torque and total work of the elbow flexors (dominant and non-dominant).

\begin{tabular}{ccccccccc}
\hline & \multicolumn{4}{c}{ PT/BW (\%) - dominant } & \multicolumn{3}{c}{ PT/BW (\%) - non-dominant } \\
\hline & PRE & POST & $15^{\text {th }}$ & $\mathbf{3 0}^{\text {th }}$ & PRE & POST & $15^{\text {th }}$ & $3^{\text {th }}$ \\
\hline Case & 10.5 & +29.52 & +77.1 & +77.2 & 15.9 & +25.16 & +30.2 & -1.9 \\
Control \#1 & 26.7 & +17.60 & -12.7 & -2.6 & 26.8 & +1.12 & -4.5 & -8.6 \\
Control \#2 & 27.8 & +10.7 & -10.1 & -2.2 & 22.5 & -0.89 & +11.0 & +20.0 \\
\hline & Total work (J) & - dominant & Total work (J) - non-dominant \\
\hline Case & PRE & POST & $15^{\text {th }}$ & $30^{\text {th }}$ & PRE & POST & $15^{\text {th }}$ & $30^{\text {th }}$ \\
\hline Control \#1 & 42.5 & +11.7 & +59.3 & +94.6 & 68.2 & +31.3 & +31.4 & +0.7 \\
Control \#2 & 160.5 & +22.6 & -18.1 & -7.5 & 170.2 & +0.18 & -22.8 & -13.2 \\
\hline & 158.5 & +19.4 & -5.4 & +0.7 & 134.1 & +2.39 & +11.9 & +12.0 \\
\hline
\end{tabular}

The data was analyzed by percentage changes from the baseline: [ $\Delta$ (POST - PRE)/100]\%; PT/BW (\%): peak torque normalized by body weight of dominant and non-dominant elbow.

During all protocols, there was neither disease relapse nor clinical intercurrences.

\section{Discussion}

To date, this is the first study to evaluate the effects of acute and chronic tDCS in a patient with DM. The tDCS was safe throughout the protocol, with no clinical adverse effects, as well as no adverse effects related to tDCS. Furthermore, in acute symptoms, it promoted increases after three sessions on the muscle torque 
and total work of dominant and non-dominant elbow flexors.

In the present study, any confounding factors were excluded, there were no changes in pharmacological treatment, and clinical evaluation was performed by a single rheumatologist. Additionally, in relation to the evaluation of the muscle torque, we tried to avoid the factors that could interfere in the values, such as pre-activation potentiation, learning effect, and physical activity level.

The initial analysis of the results in the present study corroborates studies in healthy individuals with similar experimental designs [5]. Remarkably, after three weeks, there was a significant difference in muscle torque values and total patient work compared to the Sham patients. In accordance with Hendy et al. [6], the difference was observed in the dominant limb after six weeks. Moreover, the findings of the present study are supported by previous evidence, highlighting the study by Krishnan et al. Thus showing, the tDCS was able to promote increased activation of agonist muscles of the elbow flexors excluding confounding factors as a contribution of synergists and antagonists [7].

These findings are explained possibly by the neuroplasticity induced by tDCS, the increase in neuronal excitability affects the corticospinal tract influencing the pool of motor neurons, leading to an increase in the firing frequency resulting in increased muscle activation [8].

As a limitation of the present study, we did not evaluate neurophysiological outcomes for understand the probable changes in cortical excitability and not assess the neuromuscular adaptations.

The encouraging results observed in the present study show tDCS are safe in stable DM disease, and it may be effective in maintaining muscle torque. Moreover, the present study brings new therapeutic possibilities with the potential to directly impact clinical practice, including to disease damage, such as chronic pain, fatigue, subjective muscle weakness, and others. Therefore, clinical trials with greater patient samples with different disease states and with longer intervention times are required to corroborate the present findings.

\section{Conclusion}

The present study showed that the application of transcranial direct current electrical stimulation is safe in a patient with dermatomyositis and seems to influence the ability to produce muscle strength.

\section{Funding}

Fundação de Amparo à Pesquisa do Estado de São Paulo (FAPESP) \#2019/12155-5 to RGM; Conselho Nacional de Desenvolvimento Científico e Tecnológico (CNPq) 303379/2018-9 to SKS; Faculdade de Medicina da USP to SKS.

\section{Conflicts of Interest}

The authors declare no conflicts of interest regarding the publication of this paper. 


\section{References}

[1] Lundberg, I.E., Tjärnlund, A., Bottai, M., Werth, V.P., Pilkington, C., de Visser, M., et al. (2017) European League against Rheumatism/American College of Rheumatology Classification Criteria for Adult and Juvenile Idiopathic Inflammatory Myopathies and Their Major Subgroups. Arthritis \& Rheumatology, 69, 2271-2282. https://doi.org/10.1002/art.40320

[2] Brown, L.E. (2001) ASEP: Procedures Recommendation 1: Accurate Assessment of Muscular Strength and Power. Journal of Exercise Physiology, 4, 1-21.

[3] Miller, F.W., Rider, L.G., Chung, Y.L., Cooper, R., Danko, K., Farewell, V., et al. (2001) Proposed Preliminary Core Set Measures for Disease Outcome Assessment in Adult and Juvenile Idiopathic Inflammatory Myopathies. Rheumatology (OXford), 40, 1262-1273. https://doi.org/10.1093/rheumatology/40.11.1262

[4] Bruce, B. and Fries, J.F. (2003) The Stanford Health Assessment Questionnaire: Dimensions and Practical Applications. Health and Quality of Life Outcomes, 1, 20. https://doi.org/10.1186/1477-7525-1-20

[5] Hazime, F.A., da Cunha, R.A., Soliaman, R.R., Romancini, A.C.B., Pochini, A.C., Ejnisman, B., et al. (2017) Anodal Transcranial Direct Current Stimulation (tDCS) Increases Isometric Strength of Shoulder Rotators Muscles in Handball Players. International Journal of Sports Physical Therapy, 12, 402-407.

[6] Hendy, A.M., Teo, W.P. and Kidgell, D.J. (2015) Anodal Transcranial Direct Current Stimulation Prolongs the Cross-Education of Strength and Corticomotor Plasticity. Medicine \& Science in Sports \& Exercise, 47, 1788-1797. https://doi.org/10.1249/MSS.0000000000000600

[7] Krishnan, C., Ranganathan, R., Kantak, S.S., Dhaher, Y.Y. and Rymer, W.Z. (2014) Anodal Transcranial Direct Current Stimulation Alters Elbow Flexor Muscle Recruitment Strategies. Brain Stimulation, 7, 443-450.

https://doi.org/10.1016/j.brs.2014.01.057

[8] Taylor, J.L., Amann, M., Duchateau, J., Meeusen, R. and Rice, C.L. (2016) Neural Contributions to Muscle Fatigue: From the Brain to the Muscle and Back again. Medicine \& Science in Sports \& Exercise, 48, 2294-2306. https://doi.org/10.1249/MSS.0000000000000923

[9] Lefaucheur, J.P., Antal, A., Ayache, S.S., Benninger, D.H., Brunelin, J., Cogiamanian, F., et al. (2017) Evidence-Based Guidelines on the Therapeutic Use of Transcranial Direct Current Stimulation (tDCS). Clinical Neurophysiology, 128, 56-92. https://doi.org/10.1016/j.clinph.2016.10.087

[10] Mendonca, M.E., Simis, M., Grecco, L.C., Battistella, L.R., Baptista, A.F. and Fregni, F. (2016) Transcranial Direct Current Stimulation Combined with Aerobic Exercise to Optimize Analgesic Responses in Fibromyalgia: A Randomized Placebo-Controlled Clinical Trial. Frontiers in Human Neuroscience, 10, 68. https://doi.org/10.3389/fnhum.2016.00068 